\title{
Integrating Fuzzy based Fault diagnosis with Constrained Model Predictive Control for Industrial Applications
}

\author{
Geetha Mani ${ }^{\dagger}$ and Natarajan Sivaraman*
}

\begin{abstract}
An active Fault Tolerant Model Predictive Control (FTMPC) using Fuzzy scheduler is developed. Fault tolerant Control (FTC) system stages are broadly classified into two namely Fault Detection and Isolation (FDI) and fault accommodation. Basically, the faults are identified by means of state estimation techniques. Then using the decision based approach it is isolated. This is usually performed using soft computing techniques. Fuzzy Decision Making (FDM) system classifies the faults. After identification and classification of the faults, the model is selected by using the information obtained from FDI. Then this model is fed into FTC in the form of MPC scheme by Takagi-Sugeno Fuzzy scheduler. The Fault tolerance is performed by switching the appropriate model for each identified faults. Thus by incorporating the fuzzy scheduled based FTC it becomes more efficient. The system will be thereafter able to detect the faults, isolate it and also able to accommodate the faults in the sensors and actuators of the Continuous Stirred Tank Reactor (CSTR) process while the conventional MPC does not have the ability to perform it.
\end{abstract}

Keywords: FTC, Predictive Control, Fuzzy Logic, CSTR, Takagi-Sugeno

\section{Introduction}

Process control industries operations depend widely on exceptionally automated control framework to manage progressively stringent necessities of environment sustainability, security and also profitability. Because of increasing automation components and complexity in the applications, the chemical industries face an additional problem i.e. faults in the components. Generally, faults are unavoidable and it occurs in day to day activities. In order to manage the fault, we need to employ tolerance mechanism to it. For this, it requires proper identification of faults in the process. Control algorithms need to be designed in such a way that it is capable of withstanding highly non-linear dynamics of the process under the constraint environment. Further, for a fault tolerant control it requires a master that manages the entire process and identify the faults faster. It is also mandatory to have higher switching response between the schemes i.e. when a control configuration is getting failed, the process should immediately switch over to the functioning scheme to have continuity of the process and fault tolerance capability [1].

Primarily the occurrence of the faults in the process control industries are because of sensors and actuators. Some of the noticeable soft faults are bias or drifts. So, the work of the control algorithms is to quickly detect the faults in the sensors and actuators. Further it should take necessary actions, to avoid loss in production and

$\dagger$ Corresponding Author: School of Electrical Engineering, VIT University, India. (geethamr@gmail.com)

* Dept. of Instrumentation \& Control Systems Engineering, PSG College of Technology, India.

Received: March 3, 2014; Accepted: July 5, 2016 degradation in the closed loop performance. The scope of research is inclined towards the detection of faults in realtime which is often called as online based approach.

Quantitative and qualitative are the methods to identify the fault and diagnosis it in the existing methodology. The methods are derived based on the process history and model. The qualitative method uses soft computing technique [2] whereas quantitative method utilizes estimation mechanism along with filtering $[3,6]$. Generalized likelihood ratio (GLR) based fault tolerant control system was developed by Prakash et. al. [1] for a CSTR system. The main drawback of this method is that estimation of the parameter $\theta$ would become difficult when fault ratio increases. Thus to overcome this, residues are generated to identify the fault deeply.

In the process industries, predictive algorithms are employed intensively because of its unique features like it is capable of dealing with complex process system under constraint environment. Thus by integrating the Model Predictive Controller (MPC) with the fault detection and isolation system a fault tolerant control system could be developed. MPC scheme was first employed by Maciejowski [4]. Later, a principle component based fault tolerance system was developed by Pranatyasto and Qin [5] for Fluid Catalytic Cracking (FCC) unit.

Extended Kalman Filter (EKF) and Fuzzy Decision Making (FDM) system are employed together for automatic detection of the faults in the process is the novelty work proposed in this paper. Further, in order to accommodate the faults after the identification, a FTC system is developed based on the fuzzy scheduled MPC scheme. In order to validate the functioning of the algorithm, simulation 
works were carried out using benchmark CSTR system. The sensors and actuators faults were able to identify effectively in the system. The performance of the proposed fuzzy scheduled based predictive controller is illustrated using simulation results. Further, the mathematical model of CSTR is obtained from Geetha et. al. [8].

Organization of paper is as follows: Next section of the paper discuss about the FDI technique. It is followed by description of architecture proposed for FTC in section 3. Section 4 shows the simulation results and discussion on it followed by conclusion in section 5 .

\section{Fault Detection and Isolation Technique}

Sensors and actuators are mainly prone to faults in the system. The major role of FDI system is to monitor the health of the sensor and actuators in the system. If there are any faults FDI identify it immediately then the magnitude of the faults are estimated. Kalman filter is basically used for estimation process. It utilizes the estimated residues and classifies the faults based on fuzzy decision system [7]. So, that the faults could be controlled and the system would function normally.

\subsection{Rules for fuzzy decision making system}

The rules are defined in the FDM system. Based on the number of faults rules are framed. Then the membership functions are defined for input and output variables. It is followed by mapping with the If-Then rules. Moreover, it is not mandatory to define all rules for all the cases. Only rules for computing membership function could be initialized. In this work there are only 4 rules. Therefore the fault models for 4 rule structure are defined as follows:

If Residue is FF Then Fault Index is $I_{o}$ If Residue is ACT Then Fault Index is $I_{1}$ If Residue is SEN Then Fault Index is $I_{2}$ If Residue is SIM Then Fault Index is $I_{3}$
Where, residues are the membership functions defined for fault models. This rule consists of AND operator. The advantage of using it is that it will not fired even if any of the input signals falls outside the membership function. It is usually used to integrate antecedents. The min operator will get fired and delivers output on the other side. This will be used for defuzzification process for designing fuzzy system. Input signature parameters are computed for all the process. This is given to the fuzzy classifier system that estimates the degree of certainty of all components.

\section{Proposed FTC methodology}

The block diagram of proposed FTC is shown in Fig. 1. This architecture consist of following three modules State estimator for fault detection, Fuzzy decision making system for fault isolation and MPC for optimal control and Tagaki-Sugeno Fuzzy scheduler for smooth switching of models in MPC at qualified location.

\subsection{Predictive control}

The model predicts the output of the plant by referring its past and present values. Thus depending upon the predicted output the control action is taken in the future. Further depending upon the constraints and cost function an optimum solution for control action were obtained.

Critical role is played by the model of the process (system). Based on it the control action is performed. The model should be obtained carefully so that it is able to implement and also predict the future outputs. Thereby, controllability would become feasible. The MPC elements are an objective function, a model for prediction and finally techniques to obtain the control law. MPC is formulated as constraint optimization problem at any sampling instant $\mathrm{k}$ and where the future manipulated input moves denoted as $\left\{m\left(k \mid k, m(k+1 \mid k) \ldots m\left(k+N_{p} \mid k\right)\right\}\right.$

It could be identified by minimized the objective function. The objective function used in a MPC is formulated in the form of: $\mathrm{J}=\mathrm{J}_{\mathrm{e}}+\mathrm{J}_{\Delta \mathrm{u}}$

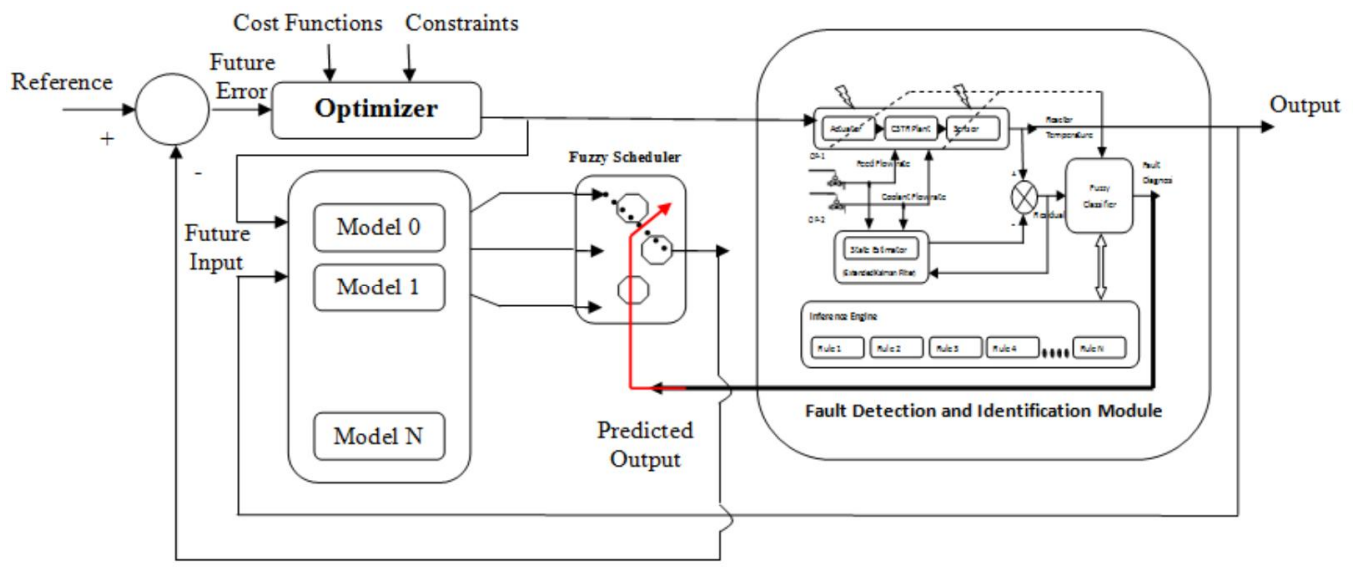

Fig. 1. Proposed FTC Architecture 


$$
\begin{aligned}
& J_{e}=\sum_{i=1}^{m} e_{f}(k+i \mid k)^{T} W_{e} e_{f}(k+i \mid k) \\
& \mathrm{J}_{\Delta \mathrm{u}}=\sum_{i=1}^{n-1} \Delta u(k+i \mid k)^{T} W_{u} \Delta u(k+i \mid k)
\end{aligned}
$$

where,

$$
\begin{aligned}
& e_{f}(k+i \mid k)=y_{r}(k+i)-\hat{y}(k+i \mid k) \\
& \Delta u(k+i \mid k)=u(k+i)-u(k+i-1 \mid k)
\end{aligned}
$$

The constraints imposed are: $\quad u^{i} \leq u(k+i \mid k) \leq u^{N}$

Where, $i \in\left[\begin{array}{ll}0 & m\end{array}\right]$. Here $m$ denoted the horizon for prediction and $n$ denotes the horizon for control, $y_{r}$ denoted set-point trajectory of future and $\hat{y}$ denotes the vector of controlled outputs.

As soon as the fault is identified by the FDI, a switching signal $s(t)$ is initiated by the controller. The process of switching is based on fuzzy fault index. It ensures that the regulatory and closed loop performance of the system is not altered. A switching signal $s(t)$ is constructed in the following equation. The rule that describes the operating point of the nonlinear system is as follows:

$$
\begin{aligned}
& \text { Rulei }(i=1: N) \\
& \begin{array}{c}
\text { If } z_{1}(k) i s M_{i, 1} \text { and }_{g}(k) i s M_{i, g} \\
\text { Then } s(t)=x(k+1)=A_{i} x(k)+B_{i} x(k) \\
\quad y(k+1)=C_{i} x(k)+D_{i} x(k)
\end{array}
\end{aligned}
$$

Where, $z_{j}(k)$ are the premise variables and $M_{i j}(k)$ are the fuzzy sets. $A_{i}, B_{i}, C_{i}$ and $D_{i}$ are known as time invariant matrices.

It is assumed in this paper that using the first principle the model could be obtained by linearizing them around different operating steady state values. By integrating all the fuzzy based linear model outputs, global behavior of the system were determined. Given an input vector $u(k)$, the global state and output of fuzzy are inferred as follows:

$$
\begin{aligned}
& x(k+1)=\sum_{i=1}^{N} h_{i}(z(k))\left[A_{i} x(k)+B_{i} u(k)\right] \\
& y(k+1)=\sum_{i=1}^{N} h_{i}(z(k))\left[C_{i} x(k)+D_{i} u(k)\right]
\end{aligned}
$$

Where grades of membership $h_{i}(z(k))$ are defined as

$$
\begin{aligned}
& h_{i}(z(k))=\frac{\mu_{i}(z(k)}{\mu(k)} \text { and } \mu_{i}(z(k))=\prod_{j=1}^{g} M_{i j} \\
& \mu(k)=\sum_{i=1}^{N} \mu_{i}(z(k))
\end{aligned}
$$

Membership grade must be

$$
h_{i}(z(k)) \in\left[\begin{array}{ll}
0 & 1
\end{array}\right] \text { and } \sum_{i=1}^{N} h_{i}(z(k))=1
$$

\section{Results and Discussions}

State space model of the CSTR process is used for all simulation process. Here with the sampling time of 1 second, prediction horizon of $\mathrm{p}=2$ and control horizon of $\mathrm{m}=1 \mathrm{MPC}$ scheme for CSTR system were developed.

Formulation of MPC involves two matrices namely weighting matrix $\left(\mathrm{W}_{\mathrm{E}}\right)$ and controller matrix $\left(\mathrm{W}_{\mathrm{U}}\right)$. It is formulated as $\mathrm{W}_{\mathrm{E}}=10 ; \mathrm{W}_{\mathrm{U}}=10$. Constrain imposed on the coolant flow rate which is a manipulated input variable is $96<u \leq 103 \mathrm{l} / \mathrm{min}$. Under fault free condition the residue signal is zero. When fault occurs, the residue i.e., diagnostic signal is no longer zero.

The residue generation and evaluation based on fuzzy decision making has discussed. Based on Fault Index identified by FDI component, the controller is reconfigured at qualified locations. FTMPC \& conventional MPC are demonstrated for different Fault conditions through simulation studies on CSTR process.

\section{Case 1: Fault free condition}

Fig. 2 shows the closed loop response when the system is fault free condition. Both conventional MPC and FTMPC tracks given set point.

\section{Case 2: Sensor fault}

In Fig. 3 the simulation results shows that the FTMPC is able to track the set point where as the conventional MPC does not, when multiplicative sensor fault is introduced at sampling 80th instant. FTMPC behaves like fault free

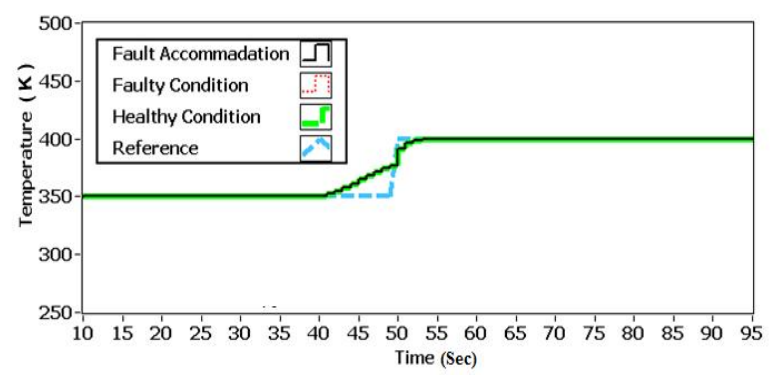

Fig. 2.Closed loop response: fault free condition

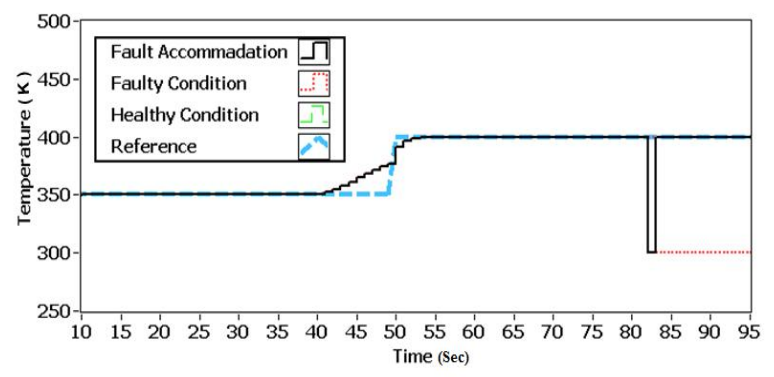

Fig. 3. Closed loop response: in the presence of sensor fault 


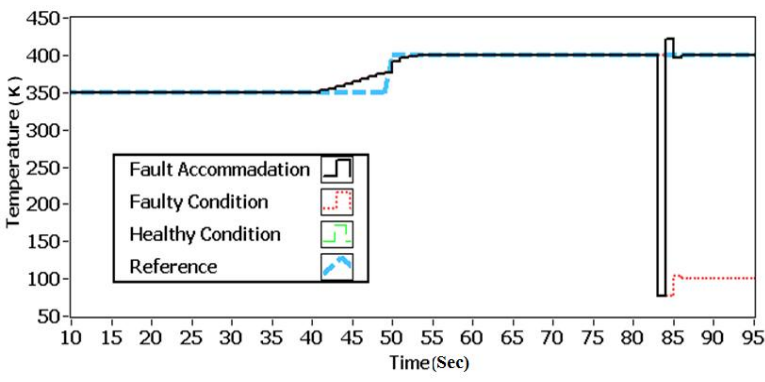

Fig. 4. Closed loop response: in the presence of both sensor and actuator fault

condition even fault has occurred.

\section{Case 3: Fault in both sensor and actuator}

The simulation result shown in Fig. 4 for the condition when both multiplicative sensor fault and actuator is introduced at 80th instant, FTMPC track the set point under fault condition like healthy mode whereas conventional MPC gives offset.

In the Figs. 2-4 shown above, Fault accommodation represents the FTMPC result. Fault condition indicates the output of conventional MPC. Healthy condition shows the output when fault free condition. Reference means set point variation.

\section{Conclusion}

Fuzzy scheduled based FTMPC scheme is the novelty proposed in this work. The proposed scheme is able to tolerate and work in the presence of sensor and actuator faults. FTC is integrated with Model Predictive control (MPC) and fuzzy based FDI is performed. Fault detection is accomplished by residue generation from extended Kalman filter. Fault Isolation is done by fuzzy decision making approach based on residue evaluation. Fault accommodation is made by smooth switching of faulty model in MPC using T-S Fuzzy scheduler at qualified location. The developed scheme is tested with a simulated version of CSTR system. The results indicate that the scheme is able to identify the faults. Further it is also able to isolate the same and tolerate the soft sensor and actuator faults simultaneously while conventional MPC fails to perform. FTMPC eliminates the offset between set point and process variable. The future scope of this work is that it could be extended to both soft and hard faults in addictive and multiplicative nature.

\section{References}

[1] Prakash J., Narasimhan Shankar, Patwardhan Sachin C.: Integrating Model Based Fault Diagnosis with
Model Predictive Control. Industrial \& Engineering Chemistry Research. vol. 44 (12), pp. 4344-4360, (2005).

[2] U. Sabura Banu and G. Uma: ANFIS based sensor fault detection for continuous stirred tank reactor. Applied Soft Comp., vol. 11, pp. 2618-2624, (2011).

[3] J.J. Gertler: A survey of model based failure detection and isolation in complex plant. IEEE Control Syst. Mag., vol. 8, pp. 3-11, (1988).

[4] Maciejowski J. M.: Modeling and Predictive Control: Enabling Technologies for reconfiguration. Annual Reviews in Control, vol. 23, pp. 13-23, (1999).

[5] T. N. Pranatyasto, S. Joe Qin.: Sensor validation and process fault diagnosis for FCC units under MPC feedback. Control engg. practice, vol. 9, pp. 877-888, (2001).

[6] Isermann, R.: Process Fault Diagnosis Based on Modeling and Estimation Methods: A survey. Automatica, vol. 20, pp. 387-404, (1984).

[7] M Geetha, Jovitha Jerome: Fuzzy expert system based sensor and actuator fault diagnosis for continuous stirred tank reactor. 2013 International Conference on Fuzzy Theory and Its Applications (iFUZZY), pp. 251-257, (2013).

[8] M Geetha, Jovitha Jerome, Arun Kumar: Critical evaluation of non-linear filter configurations for the state estimation of Continuous Stirred Tank Reactor. Applied soft computing, vol. 25, pp. 452-460, (2014).

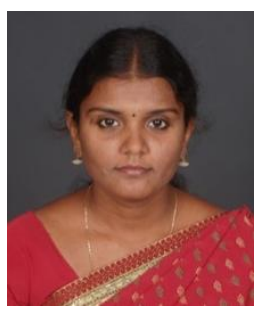

Geetha Mani received BE degree in 2004 from Madurai Kamaraj University, India and ME degree from MIT Campus, Anna University, India. She obtained $\mathrm{PhD}$ from Anna University, Chennai. She has been teaching for 9+ years at reputed institutions. Presently, she is an associate professor in School of Electrical Engineering at VIT University, Vellore, India. Her research interests include Process control, Industrial Automation, and Virtual instrumentation.

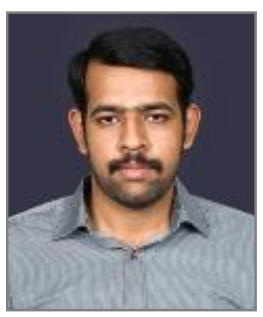

Natarajan Sivaraman received B.E. degree from Anna University, India and M.E. degree from PSG College of Technology, Anna University, India. Presently, he is an Assistant Professor in Instrumentation \& Control Systems Engineering Department at PSG College of Technology, Coimbatore, India. His research interests include embedded control systems and machine learning. 